\title{
Coronavirus disease 2019 (COVID-19) in a Chinese renal transplant recipient: a case report
}

\author{
Jian $\mathrm{Wu}^{1} \wedge$, Liangkun Xiong ${ }^{2 \wedge}$, Peng $\mathrm{Li}^{3 \wedge}$ \\ ${ }^{1}$ Department of Anesthesiology, Shengjing Hospital of China Medical University, Shenyang, China; ${ }^{2}$ Department of Hepatobiliary Surgery, Renmin \\ Hospital of Wuhan University, Wuhan, China; ${ }^{3}$ Department of Pulmonary and Critical Care Medicine, Shengjing Hospital of China Medical \\ University, Shenyang, China \\ Correspondence to: Peng Li, MD. Department of Pulmonary and Critical Care Medicine, Shengjing Hospital of China Medical University, No. 36 \\ Sanhao Street, Heping District, Shenyang 110004, China. Email: lipengcmu@163.com.
}

\begin{abstract}
Since December 2019, a novel coronavirus that caused viral pneumonia broke out and became global pandemic. Coronavirus disease 2019 (COVID-19) is caused by the SARS-CoV-2 virus. Reports on the clinical manifestations in solid organ transplant (SOT) recipients are rare. We report the clinical features and treatment of a Chinese renal transplant recipient with COVID-19. A 46-year-old Chinese woman, who had a renal transplant in 2006 due to chronic glomerulonephritis, was admitted to Renmin Hospital of Wuhan University for fever, cough, and expectoration for more than 10 days and diarrhea for 3 days. At admission, her body temperature was $38.2^{\circ} \mathrm{C}$ and pulse oxygen saturation was $96 \%$ under oxygen inhalation. There were decreased breath sounds bilaterally. Laboratory data revealed normal leucocyte count, a normal percentage of neutrophils, a normal percentage of lymphocytes, decreased lymphocyte count, elevated procalcitonin and C-reactive protein (CRP), and increased levels of urea, creatinine, and estimated glomerular filtration rate. COVID-19 was confirmed by nasopharyngeal swab and sputum which were positive for SARS-CoV-2 by real-time reverse transcription PCR (RT-PCR). Chest CT revealed multiple patchy and flake groundglass shadows in bilateral lung fields, and strip shadows in bilateral lower lobes. Treatment included antiviral (umifenovir, hydroxychloroquine), antibacterial (moxifloxacin), and other support therapies. Her symptoms, laboratory data, and chest CT showed trends of gradual improvement, while nasopharyngeal swabs were always positive for SARS-CoV-2. She was finally discharged from hospital on her 70th day of hospitalization when 2 consecutive nasopharyngeal swabs were negative for SARS-CoV-2. This is a rare report on COVID-19 in a renal transplant recipient, which can help enhance the understanding and treatment of COVID-19 in renal transplant recipients.
\end{abstract}

Keywords: Coronavirus disease 2019 (COVID-19); 2019-nCoV; SARS-CoV-2; renal transplant recipient; case report

Submitted Mar 20, 2021. Accepted for publication May 19, 2021.

doi: $10.21037 /$ tau-21-314

View this article at: http://dx.doi.org/10.21037/tau-21-314

\section{Introduction}

The World Health Organization (WHO) declared the outbreak of 2019 novel coronavirus (2019-nCOV) infected pneumonia a Public Health Emergency of International
Concern on 30 January, 2020 (1). The 2019-nCOV is also called severe acute respiratory syndrome coronavirus 2 (SARS-CoV-2) (2). As of 3 May, 2021, a total of 103,464 cases of coronavirus disease 2019 (COVID-19) have been

\footnotetext{
^ ORCID: Jian Wu, 0000-0002-3439-7435; Liangkun Xiong, 0000-0002-6596-4762; Peng Li, 0000-0002-6060-0089.
} 
confirmed in China, including 4,856 deaths (3).

Coronaviruses mainly cause respiratory tract infections, and some strains have high infectivity and mortality causing significant damage to public health, such as severe acute respiratory syndrome (SARS) and Middle East respiratory syndrome (MERS) (4). SARS and MERS in transplant recipients have been reported $(5,6)$, while research on COVID-19 in renal transplant recipients is scarce (7), and no case report about Chinese. Here, we report the clinical manifestations and treatment of a Chinese renal transplant recipient with COVID-19.

We present the following article in accordance with the CARE reporting checklist (available at http://dx.doi. org/10.21037/tau-21-314).

\section{Case presentation}

Informed written consent was obtained from the patient for publication of this report and any accompanying images. All procedures performed in studies involving human participants were in accordance with the ethical standards of the institutional and/or national research committee(s) and with the Helsinki Declaration (as revised in 2013).

\section{Main symptoms}

A 46-year-old Chinese woman, who had a renal transplant in 2006 due to chronic glomerulonephritis, was admitted to Renmin Hospital of Wuhan University complaining of fever, cough, and expectoration for more than 10 days and diarrhea for 3 days.

\section{History of present illness}

On January 22, 2020, the patient had a fever with no obvious inducement and a temperature of $37.5^{\circ} \mathrm{C}$. On January 26 , she had a fever up to $38.6^{\circ} \mathrm{C}$, with cough and expectoration. She visited Zhongshan Hospital, Wuhan where the SARS-CoV-2 nucleic acid test showed a positive result and she received antibacterial and antiviral treatment. On February 3, she had an attack of watery diarrhea. For further treatment, the patient visited Renmin Hospital of Wuhan University on February 6 and was then admitted to the hospital (February 6, 2020 was defined as day 1). It was noted that she had poor appetite without oliguria or previous contact history with COVID-19 patients.

\section{History of past illness}

The patient had a renal transplant on August 14, 2006 due to chronic glomerulonephritis and subsequently received tacrolimus and mycophenolate mofetil for antirejection maintenance therapy.

\section{Physical examination at admission}

A physical examination at admission showed a body temperature of $38.2{ }^{\circ} \mathrm{C}$, blood pressure of $111 / 74 \mathrm{mmHg}$, heart rate of 101 beats/min, respiratory rate of 18 breaths/min, and pulse oxygen saturation of $96 \%$ under $5-10 \mathrm{~L} / \mathrm{min}$ oxygen inhalation by mask. There were decreased breath sounds in bilateral lungs and no obvious abnormality in heart auscultation.

\section{Laboratory examinations}

At admission, blood examination revealed that her leucocyte count was in the normal range. Percentage of neutrophils and percentage of lymphocytes were also within the normal range. Lymphocyte count was below the normal range. The levels of procalcitonin and CRP were elevated which suggested the patient might have a bacterial infection (Table 1). Tacrolimus level was $5 \mathrm{ng} / \mathrm{mL}$. Blood gas analysis revealed arterial partial pressure of oxygen of $141.00 \mathrm{mmHg}$, arterial partial pressure of carbon dioxide of $41.0 \mathrm{mmHg}$, and a $\mathrm{pH}$ value of $7.38(5 \mathrm{~L} / \mathrm{min}$ oxygen inhalation by mask during blood sampling). Biochemical test results are shown in Table 2. Renal function test revealed urea of $10.00 \mathrm{mmol} / \mathrm{L}$, creatinine of $139.00 \mu \mathrm{mol} / \mathrm{L}$, and estimated glomerular filtration rate of $39.06 \mathrm{~mL} / \mathrm{min}$. She had hyponatremia with serum sodium of $135 \mathrm{mmol} / \mathrm{L}$ and hyperkalemia with potassium of $5.64 \mathrm{mmol} / \mathrm{L}$. There was no proteinuria or hematuria. The coagulation function test showed fibrinogen levels were increased to $5.70 \mathrm{~g} / \mathrm{L}$, whereas the levels of prothrombin time, activated partial thromboplastin time, thrombin time, D-dimer, and fibrinogen degradation products were in the normal range. Antibody IgM detection of cytomegalovirus and Epstein-Barr virus were negative. COVID-19 was confirmed on February 8, 2020 by nasopharyngeal swab and sputum, which were positive for SARS-CoV-2 by RT-PCR.

\section{Imaging examinations}

On February 8, 2020, chest CT revealed multiple patchy 
Table 1 Clinical laboratory results of the patient

\begin{tabular}{|c|c|c|c|c|c|c|c|c|c|}
\hline Examination items & Day $1^{*}$ & Day 3 & Day 12 & Day 21 & Day 29 & Day 39 & Day 50 & Day 66 & Normal range \\
\hline \multicolumn{10}{|l|}{ Blood, routine } \\
\hline Leucocytes $\left(\times 10^{9}\right.$ per $\left.\mathrm{L}\right)$ & 4.60 & 5.84 & 9.13 & 12.27 & 12.40 & 11.46 & 9.21 & 10.72 & $3.5-9.5$ \\
\hline Neutrophils (\%) & 68.70 & 53.40 & 58.00 & 62.70 & 64.00 & 61.80 & 61.00 & 63.60 & $50-70$ \\
\hline Lymphocytes ( $\times 10^{9}$ per L) & 0.97 & 1.99 & 2.85 & 3.28 & 2.99 & 3.34 & 2.42 & 3.04 & $1.1-3.2$ \\
\hline Platelet $\left(\times 10^{9}\right.$ per $\left.\mathrm{L}\right)$ & 369.00 & 416.00 & 385 & 318 & 259.00 & 207.00 & 317 & 316.00 & $125-350$ \\
\hline C-reactive protein (CRP) (mg/L) & 38.7 & 5.1 & $<5$ & $<5$ & $<5$ & $<5$ & $<5$ & $<5$ & $0-10$ \\
\hline Procalcitonin (ng/mL) & 0.114 & & & 0.026 & & & 0.062 & 0.054 & $<0.1$ \\
\hline
\end{tabular}

*, February 6, 2020 was defined as day 1.

Table 2 Biochemical parameters of the patient

\begin{tabular}{|c|c|c|c|c|c|c|c|c|c|}
\hline Main parameters & Day $1^{*}$ & Day 4 & Day 6 & Day 21 & Day 29 & Day 39 & Day 50 & Day 66 & Normal range \\
\hline Albumin, g/L & 37.60 & 32.80 & 32.60 & 37.70 & 41.20 & 38.00 & 41.20 & 41.00 & $40-55$ \\
\hline Albumin/globulin ratio & 1.53 & 1.58 & 1.48 & 1.56 & 1.65 & 1.51 & 1.67 & 1.91 & $1.2-2.4$ \\
\hline Alanine aminotransferase, U/L & 21.00 & 12.00 & 9.00 & 13.00 & 28.00 & 31.00 & 55.00 & 29.00 & $7-40$ \\
\hline Alkaline phosphatase, U/L & 114.00 & 71.00 & 68.00 & 87.00 & 127.00 & 124.00 & 150.00 & 149.00 & $35-100$ \\
\hline$\gamma$-glutamyltransferase, $U / L$ & 173.00 & 101.00 & 87.00 & 113.00 & 158.00 & 155.00 & 192.00 & 183.00 & $7-45$ \\
\hline Lactate dehydrogenase, U/L & 218.00 & 167.00 & 157.00 & 139.00 & 201.00 & 182.00 & 185.00 & & $120-250$ \\
\hline Urea, mmol/L & 10.00 & 7.92 & 7.40 & 7.80 & 9.60 & 10.46 & 11.79 & 11.90 & $2.6-7.5$ \\
\hline Glucose, mmol/L & 7.87 & 6.55 & 7.55 & 4.23 & 3.87 & 4.59 & 4.44 & 4.70 & $3.9-6.1$ \\
\hline eGFR, $\mathrm{mL} / \mathrm{min}$ & 39.06 & 50.71 & 50.17 & 51.83 & 51.27 & 50.17 & 50.17 & & $>90$ \\
\hline Potassium $\left(\mathrm{K}^{+}\right), \mathrm{mmol} / \mathrm{L}$ & 5.64 & 4.59 & 4.52 & 4.70 & 5.02 & 4.28 & 4.26 & 4.25 & $3.5-5.3$ \\
\hline Sodium $\left(\mathrm{Na}^{+}\right), \mathrm{mmol} / \mathrm{L}$ & 135.00 & 146.00 & 147.00 & 141.00 & 141.00 & 145.00 & 143.00 & 141.00 & $137-147$ \\
\hline
\end{tabular}

*, February 6, 2020 was defined as day 1; eGFR, estimated glomerular filtration rate.

and flake ground-glass shadows in bilateral lung fields, and strip shadows in bilateral lower lobes (Figure 1A).

\section{Final diagnosis}

Our final diagnosis was moderate COVID-19 in a Chinese renal transplant recipient.

\section{Treatment}

The treatment regimen for this patient included antiviral, antibacterial, and other support treatment. For antiviral treatment, she received umifenovir $(0.2 \mathrm{~g}$ tid, po $)$ and hydroxychloroquine sulfate tablets $(0.2 \mathrm{~g}$ bid, po). For antibacterial treatment, she received moxifloxacin hydrochloride and sodium chloride injection $(0.4 \mathrm{~g} \mathrm{qd}$, 
iv drop). For support treatment, she received compound methoxyphenamine hydrochloride capsules (2 capsules bid po; each capsule contained methoxyphenamine hydrochloride $12.5 \mathrm{mg}$, narcotine $7 \mathrm{mg}$, aminophylline $25 \mathrm{mg}$, and chlorphenamine maleate $2 \mathrm{mg}$ ) to relieve the cough, and eucalyptol, limonene, pinene enteric soft capsules $(0.3 \mathrm{~g}$ tid po), and acetylcysteine effervescent tablets ( $0.6 \mathrm{~g}$ bid po) to help with expectoration. Additionally, she received Bailing capsules (a Chinese patent medicine) $(2 \mathrm{~g}$ tid po) to protect renal function, Ganjie Bingmei tablets (a traditional Chinese medicine) ( $0.4 \mathrm{~g}$ tid po) to treat acute pharyngitis, and live bacillus licheniformis capsules $(500 \mathrm{mg}$ tid po) to regulate dysbacteriosis.

\section{Outcome and follow-up}

On day 3 , she was afebrile, and her cough had subsided. On day 7 , she had a fever again, with a temperature of $37.6^{\circ} \mathrm{C}$. Biochemical tests revealed urea of $7.40 \mathrm{mmol} / \mathrm{L}$, creatinine of $113.00 \mu \mathrm{mol} / \mathrm{L}$, estimated glomerular filtration rate of $50.17 \mathrm{~mL} / \mathrm{min}$, and normal levels of serum sodium and potassium, which indicated renal function was improved after treatment. In the next 4 days, her temperature fluctuated between $36.0-38.5^{\circ} \mathrm{C}$. Subsequently, her body temperature remained normal and the patient maintained a good general condition with occasional cough, and serum procalcitonin and CRP remained at normal levels.

After admission, her chest CT showed a trend of gradual improvement. The chest CT on February 8, 2020 (Figure 1A) showed viral pneumonia. During hospitalization, 3 more chest CT examinations were performed on February 18 (Figure 1B), February 26 (Figure 1C), and March 7 (Figure 1D), indicating that the patient's pneumonia gradually improved.

However, nasopharyngeal swab, sputum, blood, stool, and urine were still positive for SARS-CoV-2 repeatedly by RT-PCR. By day 47, blood and sputum were negative for SARS-CoV-2, while nasopharyngeal swab and stool were still positive (Table 3). She was finally discharged from hospital on day 70 as she had 2 consecutive nasopharyngeal swabs that were negative for SARS-CoV-2, which met the discharge criteria drafted by the National Health Commission of the People's Republic of China (8). During hospitalization, the patient received 13 days of umifenovir, 7 days of hydroxychloroquine sulfate, and 8 days of moxifloxacin hydrochloride in total. So far, the patient has remained in good condition over one year of follow-up (the last follow-up on 22 Apr 2020).

\section{Discussion}

As the situation of COVID-19 varies greatly between and within countries, it is recommended that solid organ transplant (SOT) follow the guidelines, policies and procedures established by national authorities, as well as local and institutional policies. At present, the main prevention strategy for SOT is to avoid contact with COVID-19 (9).

Reports regarding COVID-19 in immunocompromised patients are rare. We report a case of COVID-19 in a Chinese renal transplant recipient. The COVID-19 diagnosis of this patient was based on her clinical symptoms, chest CT, and the positive detection of SARS-CoV-2 in nasopharyngeal swabs and sputum by RT-PCR on day 3 . The main clinical symptoms of this case were fever, cough, expectoration, and diarrhea, consistent with previous reports on COVID-19 in China $(10,11)$. Meanwhile, the routine blood test on admission revealed lymphopenia with normal leucocyte count, elevated CRP, and high procalcitonin levels, which suggested viral infection combined with bacterial infection. Besides blood, sputum, and nasopharyngeal swab, viral detection in her stool specimen was also positive for SARS-CoV-2, which might partially explain why she had diarrhea. This indicated that the digestive system might be another transmission pathway of SARS-CoV-2. However, the definite mechanisms of SARS-CoV-2-induced digestive symptoms are still unknown (12).

Umifenovir is an antiviral drug which is available for the treatment of influenza in Russia and China. It can produce antiviral effects against influenza $\mathrm{A}$ and $\mathrm{B}$ virus, adenovirus, respiratory syncytial virus, and coronavirus $(13,14)$. The duration of umifenovir treatment in this case was 13 days, while it was recommended by the National Health Commission of the People's Republic of China that umifenovir therapy should not exceed 10 days (8). During antiviral treatment with umifenovir in this patient, no obvious side effects were found, and after 11 days of umifenovir therapy, her temperature dropped to normal. Therefore, umifenovir may be used according to the 
A

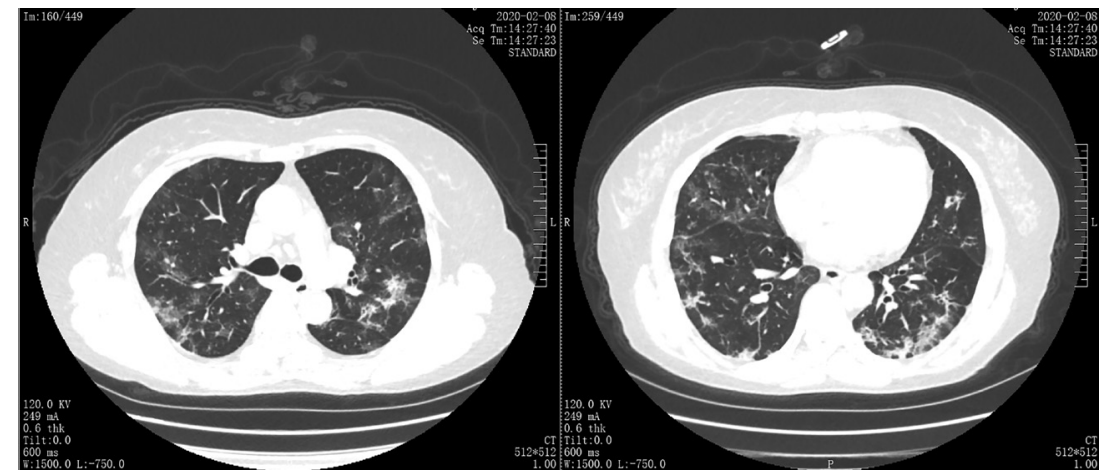

B

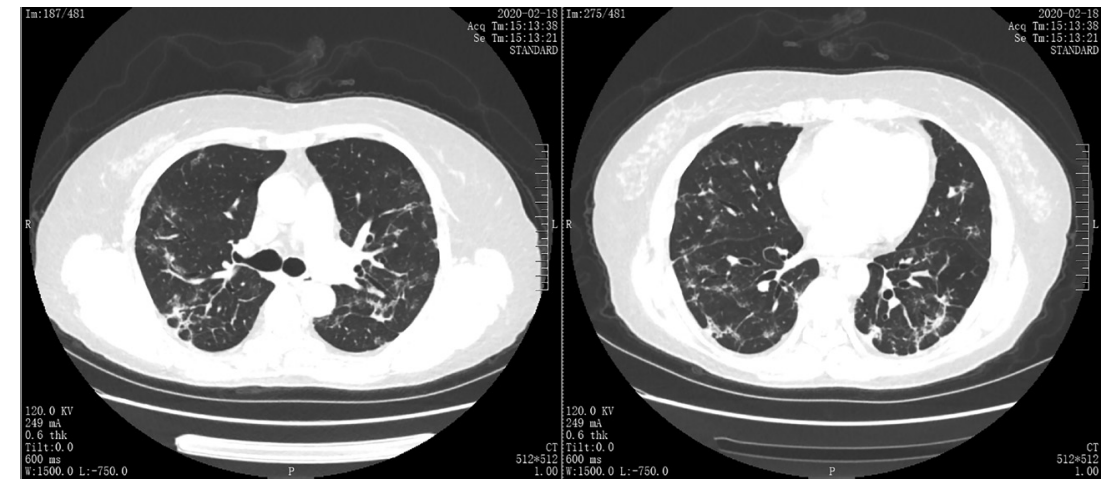

C

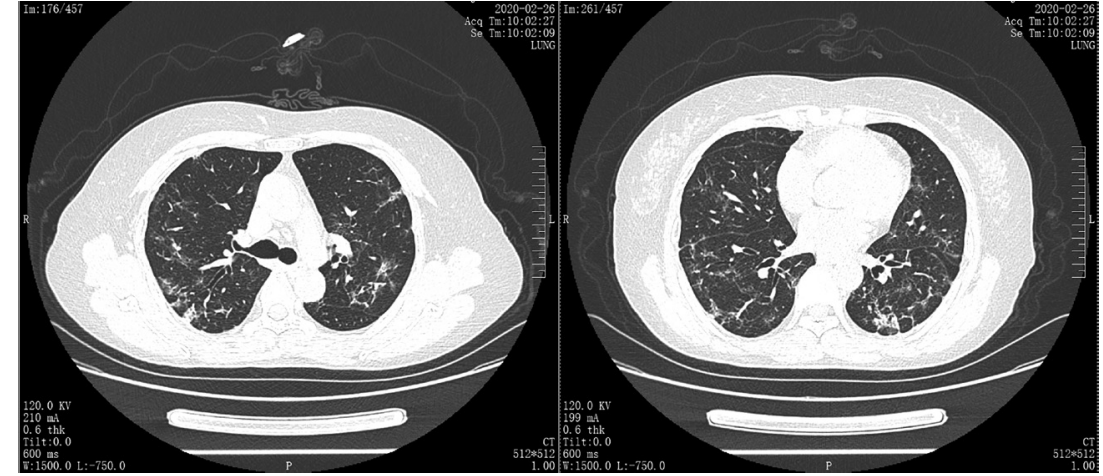

D

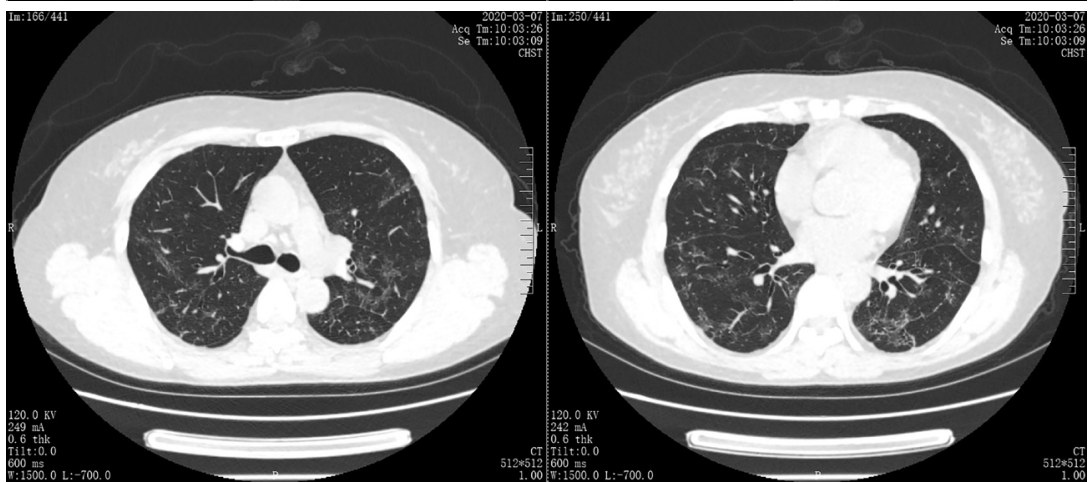

Figure 1 Results of 4 chest CT examinations during hospitalization (the same levels were selected for comparison). (A) CT showed multiple patchy and ground glass opacities in both lungs, and strip shadows in the lower lobes (taken on Feb 8, 2020). A diagnosis of viral pneumonia was obtained; (B) multiple patchy and ground glass opacities and strip shadows were found in both lungs (on Feb 18, 2020). Compared with (A) the images were improved; (C) multiple patchy ground glass opacities, and grid and streak shadows were seen in both lungs (on Feb 26, 2020). Compared with (B) the images were improved; (D) multiple patchy ground glass opacities, and grid and streak shadows were seen in both lungs (on Mar 7, 2020). Compared with (C), the images were improved. 


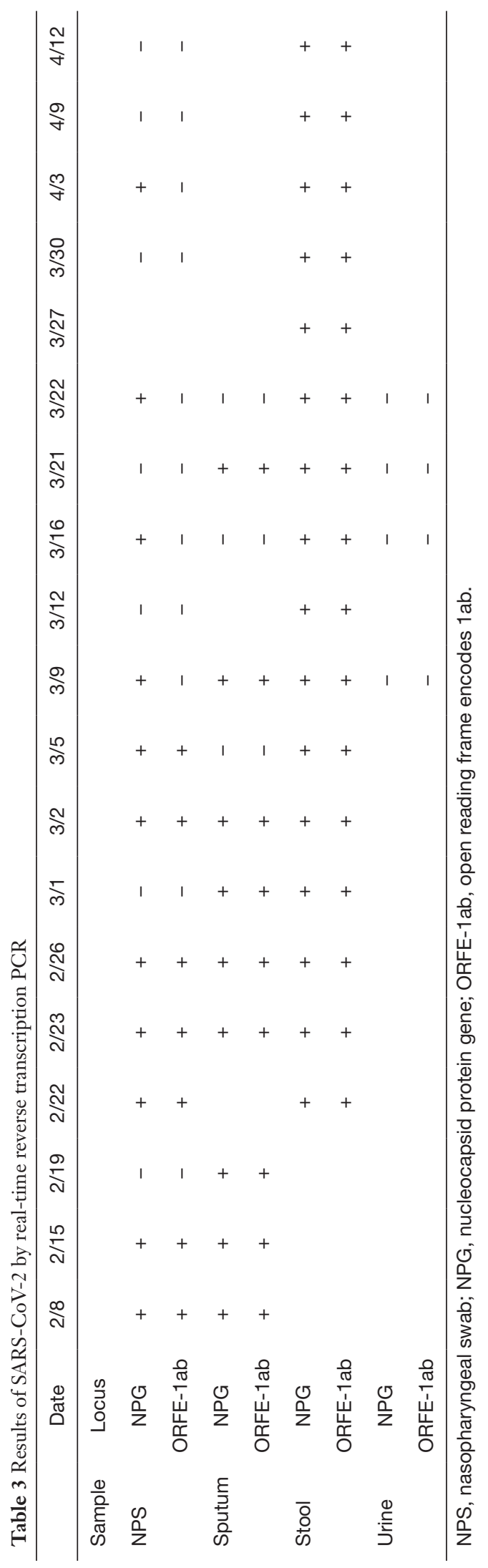

patient's symptoms or condition and it may not be necessary to limit treatment to 10 days. It was notable that this patient was young, stable, 14 years post-transplant, and had no relevant comorbidities other than being immunosuppressed for the transplant. These features were probably relevant to the positive outcome. At present, discontinuation of antiproliferative and antimetabolic drugs and continued use of steroids and calcineurin inhibitors (CNIs) are generally accepted treatment strategies for patients with COVID-19 infection after organ transplantation (15). We don't have any drugs that can reduce the replication of COVID-19 and eliminate it completely. More research is needed to provide a reference for antiviral therapy for COVID-19.

In the guidelines for the diagnosis and treatment of novel coronavirus infection by the National Health Commission (Trial Version 7), chloroquine phosphate is recommended (8). However, we used hydroxychloroquine sulfate for antiviral treatment of this patient at that time. Chloroquine phosphate, a 4-aminoquinoline, is widely used to treat malaria. Hydroxychloroquine sulfate is its derivative, which is the preferred treatment for rheumatoid arthritis, lupus erythematosus, and sarcoidosis due to its minimal ocular toxicity (16). A recent in vitro study showed that hydroxychloroquine has a more potent inhibitory action on novel coronavirus than chloroquine (17). It has been shown that chronic hydroxychloroquine protects the kidneys of mice suffering from systemic lupus erythematosus (18). Moreover, hydroxychloroquine is valuable in diabetic patients as it can prevent insulin degradation, inhibit gluconeogenesis, and enhance glucose utilization (19). The patient in this case had mild renal dysfunction and hyperglycemia, so it was more suitable to use hydroxychloroquine sulfate than chloroquine phosphate for antiviral therapy. During hydroxychloroquine sulfate treatment in this case, there was only mild gastrointestinal adverse reactions which were tolerable, and there was no significant change in renal function.

Bailing capsules are a Chinese patent medicine which can be used to treat lupus nephritis. It can improve immune function, and has anti-inflammatory, anti-hypoxia, and antitumor effects. It can also protect hepatic and renal function $(20,21)$. Bailing capsules can effectively prevent allograft rejection, stimulate hematopoiesis, and reduce infection occurrence, thereby being the perfect immunoregulators (22). We used Bailing capsules in this patient, which might have been associated with her gradually improved renal function. 
Hence, traditional Chinese medicine treatment may play an important role in renal transplant recipients with COVID-19.

In a retrospective study on COVID-19, it was found that the median hospital stay was 10 days in recovered patients (23). However, the hospital stay was 70 days in this case. The longer hospitalization period may be due to the long-term use of immunosuppressive drugs which might lead to decreased immunological function and delayed virus clearance. A previous study on immunocompromised SARS patients suggested that immunosuppressant treatment should remain unchanged to prevent renal allograft rejection (24). It is still unknown how immunosuppression may affect the course of COVID-19. Two reports on immunosuppressive patients with COVID-19 proposed reduction or withdrawal of immunosuppressants $(25,26)$. Recently, Minotti et al. found that immunosuppressive patients with COVID-19 could have a favorable disease course, in comparison with normal people. This could have been due to the possible protective effect produced by the decreased immune reaction, which determined the milder disease manifestations (27). In this case, immunosuppressive agents were not reduced during the treatment of COVID-19. The clinical symptoms and renal function of this patient improved after treatment, which suggested that the treatment could be effective with prevention of renal allograft rejection. Indeed, the patient maintained a good condition with normal temperature after 11 days in hospital, and the CT scan was almost clear 1 month after admission (Figure 1D). The length of stay was influenced by the longer time to clear the virus, but not to clinical recovery. Hence, immunosuppressive treatment may be helpful to limit the inflammatory injury of COVID-19, while virus clearance may be prolonged due to the reduced immune response, which remind that we should strengthen the screening SARS-Cov2. Further studies will be needed to clarify the effect of immunosuppression on COVID-19.

\section{Conclusions}

We report COVID-19 in a Chinese renal transplant recipient. It may provide new ideas for the understanding and treatment of COVID-19 in renal transplant recipients. In future, further data are required in order to enhance the understanding and treatment of COVID-19 in renal transplant recipients.

\section{Acknowledgments}

Funding: None.

\section{Footnote}

Reporting Checklist: The authors have completed the CARE reporting checklist. Available at http://dx.doi.org/10.21037/ tau-21-314

Conflicts of Interest: All authors have completed the ICMJE uniform disclosure form (available at http://dx.doi. org/10.21037/tau-21-314). The authors have no conflicts of interest to declare.

Ethical Statement: The authors are accountable for all aspects of the work in ensuring that questions related to the accuracy or integrity of any part of the work are appropriately investigated and resolved. Informed written consent was obtained from the patient for publication of this report and any accompanying images. All procedures performed in studies involving human participants were in accordance with the ethical standards of the institutional and/or national research committee(s) and with the Helsinki Declaration (as revised in 2013).

Open Access Statement: This is an Open Access article distributed in accordance with the Creative Commons Attribution-NonCommercial-NoDerivs 4.0 International License (CC BY-NC-ND 4.0), which permits the noncommercial replication and distribution of the article with the strict proviso that no changes or edits are made and the original work is properly cited (including links to both the formal publication through the relevant DOI and the license). See: https://creativecommons.org/licenses/by-nc-nd/4.0/.

\section{References}

1. WHO. Statement on the second meeting of the International Health Regulations (2005) Emergency Committee regarding the outbreak of novel coronavirus (2019-nCoV). [Internet]. Geneva: WHO; 2020. Accessed on 31 January 2020. Available online: https://www.who.int/ news-room/detail/30-01-2020-statement-on-the-secondmeeting-of-the-international-health-regulations-(2005)emergency-committee-regarding-the-outbreak-of-novel- 
coronavirus-(2019-ncov)

2. Coronaviridae Study Group of the International Committee on Taxonomy of Viruses. The species Severe acute respiratory syndrome-related coronavirus: classifying 2019-nCoV and naming it SARS-CoV-2. Nat Microbiol 2020;5:536-44.

3. WHO. Coronavirus disease (COVID-2019) situation reports. 2020. Available online: https://www.who.int/ emergencies/diseases/novel-coronavirus-2019/situationreports. Accessed 3 May 2021.

4. Gralinski LE, Menachery VD. Return of the Coronavirus: 2019-nCoV. Viruses 2020;12:135.

5. Kumar D, Tellier R, Draker R, et al. Severe acute respiratory syndrome (SARS) in the liver transplant recipient and guidelines for donor SARS screening. Am J Transplant 2003;3:977-81.

6. AlGhamdi M, Mushtaq F, Awn N, et al. MERS CoV infection in two renal transplant recipients: case report. Am J Transplant 2015;15:1101-4.

7. Tzukert K, Abel R, Mor Yosef Levi I, et al. The Challenge of Treating Kidney Transplant Recipients Infected with COVID-19: Report of the First Cases in Israel. Isr Med Assoc J 2020;22:602-4.

8. National Health Commission of the People's Republic of China. Guidelines for the diagnosis and treatment of novel coronavirus (2019-nCoV) infection by the National Health Commission (Trial Version 7). Available online: http://www.gov.cn/zhengce/zhengceku/2020-03/04/ content_5486705.htm

9. The Transplantation Society. Guidance on Coronavirus Disease 2019 (COVID-19) for Transplant Clinicians. Updated 1 March 2021. Available online: https://tts.org/ index.php?option=com_content $\&$ view=article $\& i d=749 \&$ It emid $=140$

10. Chen N, Zhou M, Dong X, et al. Epidemiological and clinical characteristics of 99 cases of 2019 novel coronavirus pneumonia in Wuhan, China: a descriptive study. Lancet 2020;395:507-13.

11. Huang C, Wang Y, Li X, et al. Clinical features of patients infected with 2019 novel coronavirus in Wuhan, China. Lancet 2020;395:497-506.

12. Gu J, Han B, Wang J. COVID-19: Gastrointestinal manifestations and potential fecal-oral transmission. Gastroenterology 2020;158:1518-9.

13. Brooks MJ, Burtseva EI, Ellery PJ, et al. Antiviral activity of arbidol, a broad-spectrum drug for use against respiratory viruses, varies according to test conditions. J Med Virol 2012;84:170-81.

14. Kiselev OI, Maleev VV, Deeva EG, et al. Clinical efficacy of arbidol (umifenovir) in the therapy of inflfluenza in adults: Preliminary results of the multicenter double-blind randomized placebo-controlled study ARBITR. Ter Arkh 2015;87:88-96.

15. Kędzierska-Kapuza K, Zielińska D, Matejak-Górska M, et al. The Course of SARS-CoV-2 in a Patient After a Recent Kidney Transplant: A Literature Review on COVID-19 Therapy. Transplant Proc 2021. doi: 10.1016/ j.transproceed.2021.03.008.

16. Weiss JS. Antimalarial medications in dermatology. A review. Dermatol Clin 1991;9:377-85.

17. Yao X, Ye F, Zhang M, et al. In Vitro Antiviral Activity and Projection of Optimized Dosing Design of Hydroxychloroquine for the Treatment of Severe Acute Respiratory Syndrome Coronavirus 2 (SARS-CoV-2). Clin Infect Dis 2020;71:732-9.

18. Gómez-Guzmán M, Jiménez R, Romero M, et al. Chronic hydroxychloroquine improves endothelial dysfunction and protects kidney in a mouse model of systemic lupus erythematosus. Hypertension 2014;64:330-7.

19. Quatraro A, Consoli G, Magno M, et al. Hydroxychloroquine in decompensated, treatmentrefractory noninsulin-dependent diabetes mellitus. A new job for an old drug? Ann Intern Med 1990;112:678-81.

20. $\mathrm{Xu} \mathrm{H}, \mathrm{Li}$ S. Pharmacological effects of Bailing capsule and its application in lung disease research. Zhongguo Zhong Yao Za Zhi 2010;35:2777-81.

21. Liu LJ, Ma SY, Yuan BR. The pharmacological effects and clinical application of Bailing capsule. Chinese Traditional Patent Medicine 2004;26:493-6.

22. Wang W, Zhang XN, Yin H, et al. Effects of Bailing capsules for renal transplant recipients: a retrospective clinical study. Chin Med J (Engl) 2013;126:1895-9.

23. Wang D, Hu B, Hu C, et al. Clinical Characteristics of 138 Hospitalized Patients With 2019 Novel CoronavirusInfected Pneumonia in Wuhan, China. JAMA 2020;323:1061-9.

24. Chiu MC. Suggested management of immunocompromized kidney patients suffering from SARS. Pediatr Nephrol 2003;18:1204-5.

25. Guillen E, Pineiro GJ, Revuelta I, et al. Case report of COVID-19 in a kidney transplant recipient: Does immunosuppression alter the clinical presentation? Am J 
Transplant 2020;20:1875-8.

26. Zhu L, Xu X, Ma K, et al. Successful recovery of COVID-19 pneumonia in a renal transplant recipient with long-term immunosuppression. Am J Transplant 2020;20:1859-63.

27. Minotti C, Tirelli F, Barbieri E, et al. How is

Cite this article as: $\mathrm{Wu} \mathrm{J}$, Xiong L, Li P. Coronavirus disease 2019 (COVID-19) in a Chinese renal transplant recipient: a case report. Transl Androl Urol 2021;10(5):2140-2148. doi: $10.21037 /$ tau-21-314 immunosuppressive status affecting children and adults in SARS-CoV-2 infection? A systematic review. J Infect 2020;81:e61-6.

(English Language Editor: C. Betlazar-Maseh) 